\title{
Сергей Точилин
}

\section{ПРОИЗВОДИТЕЛЬНОСТЬ RESTҒUІ И SОАР РНР WЕВ-СЕРВИСОВ ПРИ ПОИСКЕ В ДАННЫХ МУSQL}

\begin{abstract}
Актуальность темы исследования. При построении распределенных компьютерных систем широко используется сервис-ориентированная архитектура (СОА). Для практической реализации систем с СОА применяют технологии Web-сервисов. Одним из основных требований предъявляемых к Web-сервисам является производительность функиионирования, которая зависит от их программной реализации.

Постановка проблемы. Wеb-сервисы используются для доступа к информачии, которая хранится в базах данных СУБД, размещенных на Web-узлах.

В этой связи актуальной является проблема выбора оптимального программного обеспечения для реализации информационного Web-сервиса с оперативным доступом к данным.

Анализ последних исследований и публикаций. При доступе к данным Web-сервисы используют запросы к СУБД, которые, как правило, реализуют в виде команд SQL.

Для повышения эффективности работы Web-приложений, которые работают с данными, вместо команд SQL применяют хранимые процедуры и функиии СУБД.

Выделение неисследованных частей общей проблемы. Исследований производительности RESTful и SOAP PHP Web-сервисов, которые при доступе к данным MySQL с различным объемом хранимой информации в одном режиме функиионирования применяли команды $S Q L$, в другом-хранимые процедуры, не проводилось.

Постановка задачи. Определить и проанализировать производительность RESTful и SOAP PHP Web-сервисов, которые используют команды SQL и функиионально подобные хранимые прочедуры при поиске в данных MySQL, для выбора оптимального программного обеспечения Web-служб, работающих с СУБД MySQL.

Изложение основного материала. Как выяснилось, использование RESTful и SOAP PHP Web-сервисами при запросах к MySQL на поиск в данных хранимых прочедур, вместо команд SQL, снижало их производительность.

В тоже время, при поиске в исследованных объемах данных $V \leq 2$ МБайт с применением в запросах к MySQL, как команд SQL, так и хранимых прочедур, RESTful Web-сервис был более производительным, чем SOAP PHP сервис.

Выводы статьи. Установлены особенности производительности RESTful и SOAP PHP сервисов при поиске в данных MySQL с использованием, как команд SQL, так и хранимых процедур.

При поиске в данных MySQL RESTful Web-сервис во всех режимах функиионирования был более производительный чем SOAP PHP сервис.

Ключевые слова: производтельность; SOAP; RESTful; Web-сервис; MySQL.
\end{abstract}

Постановка проблемы. При построении современных распределенных компьютерных систем широко используется сервис-ориентированная архитектура (СОА). Для практической реализации систем с COA применяют технологии Web-сервисов (Web-служб) [1-3].

Web-сервисы предоставляют Web-услуги в рамках слабосвязанных Webприложений. К таким услугам, в частности, относятся услуги доступа к информации, которая хранится в базах данных (БД), созданных и поддерживаемых определенной системой управления БД (СУБД). При этом для доступа к данным используют запросы к соответствующей СУБД, которые, как правило, реализуют на структурированном языке запросов (Structured Query Language - SQL) в виде команд SQL.

$\mathrm{B}$ тоже время для повышения эффективности работы Web-приложений, которые работают с данными, вместо команд SQL применяют хранимые процедуры и функции СУБД [4].

В соответствии с [4] их использование, в частности, позволяет:

- Обеспечить дополнительную защиту данных СУБД.

- Минимизировать изменения кода приложения при изменении структуры БД.

- Уменьшить сетевой трафик, обусловленный обменом информации с сервером СУБД.

- Инкапсулировать логику работы с данными.

- Повысить транспортабельность приложений для работы с СУБД.

Однако при использовании хранимых процедур и функций может измениться производительность Web-приложений, работающих с информацией.

() Точилін С. Д., 2017 
TECHNICAL SCIENCES AND TECHNOLOGIES

В тоже время одним из основных требований, предъявляемых к Web-сервисам, является оперативность предоставления Web-услуг, которая зависит от того, каким образом сервис программно реализуется $[5 ; 6]$.

В этой связи актуальной является задача выбора оптимального программного обеспечения для реализации информационного Web-cервиса, которая тесно связана с задачей выбора или разработки программы-тестера, определяющей его производительность [6].

Анализ последних исследований и публикаций. Язык программирования РНР широко применяется для разработки распределенных приложений. При этом программные средства РНР являются эффективной платформой для СОА. С ее помощью разрабатываются Web-сервисы, поддерживающие различные протоколы и технологии [7]. В частности, службы на основе РНP и протокола SOAP, которые предоставляют Web-услуги в виде XML-сообщений.

Современные распределенные приложения с COA широко используют RESTful Webсервисы [7; 8], которые представляют удаленные ресурсы с помощью НТТР-протокола.

К отдаленным ресурсам, можно отнести и отдельные записи, которые хранятся в БД и являются доступными для сервиса. В тоже время, данные таких БД, клиент RESTful Web-сервиса может получать, добавлять, изменять и удалять, используя для этого HTTP-методы GET, PUT, POST и DELETE, соответственно, при обращении Webприложения к СУБД.

Платформа РНР имеет интерфейс программирования приложений достаточный для разработки RESTful Web-сервисов. Особенности их реализации на основе PHP API pacсматриваются в [7; 8].

Web-сервисы, широко используются для доступа к информации, которая хранится в БД СУБД, размещенных на Web-узлах. При этом в соответствии с [9] запросы к СУБД на поиск в данных являются наиболее важным типом запросов к информационному Web-узлу.

Как известно, к наиболее распространенным и производительным СУБД, которые распространяют на условиях открытых исходных кодов, относят MySQL [10]. При этом, при работе с данными MySQL применяют как команды SQL, так и хранимые процедуры [10].

Особенности производительности XML-RPC PHP Web-сервисов, использующих при поиске в различных объемах данных MySQL команды SQL, были изучены нами ранее в работе [6]. В тоже время подобных исследований для RESTful и SOAP PHP Web-сервисов не было проведено.

Кроме того не было проведено исследований производительности RESTful и SOAP PHP сервисов при доступе к данным MySQL с применением хранимых процедур.

Цель статьи. Цель данной работы - исследовать особенности производительности RESTful и SOAP PHP Web-сервисов, которые используют команды SQL и функционально подобные хранимые процедуры при поиске в данных MySQL, для выбора оптимального программного обеспечения Web-служб, работающих с СУБД MySQL. При этом для определения производительности сервисов предполагалось также разработать программу-тестер с простым интерфейсом пользователя.

Изложение основного материала. Для решения поставленной задачи было создано два варианта распределенных приложений, которые в своем составе имели одинаковую клиентскую часть (программа-тестер), и функционально подобные Web-cервисы (RESTful и SOAP), разработанные на основе PHP API.

Также были подготовлены объекты исследования - 7 таблиц (Data1 - Data7) БД СУБД MySQL, которые имели тип MyISAM. Как известно, БД MySQL с таблицами MyISAM обеспечивают высокую производительность операций поиска и чтения данных [11]. 
Кроме того, объекты исследования Data1 - Data7 имели одинаковую структуру полей (два поля - ключевое и данных), но разный объем хранимой информации $V$, в которой осуществлялся поиск (от 200 КБайт до 2 Мбайт, шаг - 300 КБайт).

Для определения и анализа производительности RESTful и SOAP PHP Webсервисов мы определяли их время отклика $\tau$ (время, которое затрачивает сервис на выполнение запроса пользователя).

Согласно [9], для определения $\tau$ можно использовать выражение: $\tau=\mathrm{T}-t$, где $\mathrm{T}-$ полное время выполнения запроса в распределенном приложении; $t$ - время обдумывания пользователя (среднее время, которое проходит между получением пользователем ответа на свой запрос и отправлением нового запроса).

Определение времени отклика $\tau$ Web-сервисов осуществлялось нами с помощью компактной программы-тестера STochno, разработанной для решения поставленной в данной работе задачи. С этой целью она получала значения Т и $t$, при последовательном выполнении распределенным приложением двух подобных алгоритмов.

Первый из них состоял из такой последовательности действий:

1.В клиентском приложении зафиксировать время начала работы распределенного приложения.

2. Выполнить пп. 3-8 определенное число раз (значение числа определяет пользователь).

3. Сформировать параметры запроса к Web-сервису.

4. Отправить из клиентского приложения запрос.

5. В программной реализации Web-сервиса получить параметры запроса клиента.

6. С помощью команды SQL или хранимой процедуры выполнить запрос к СУБД на поиск в данных с использованием параметров из п. 3.

7. Отправить результат поиска клиенту.

8. Получить в клиентском приложении результат поиска в данных.

9. Зафиксировать время.

10. Определить время работы распределенного приложения и сохранить его значение.

Второй алгоритм представлял собой сокращенную версию первого (без выполнения пп. 4-8).

При этом запрос к каждому сервису состоял из имени таблицы данных БД MySQL, параметра поиска в ней, который в ходе эксперимента менял значение случайным образом от 1 до максимально возможного для каждого объекта исследования, а также указателя на тип обращения к СУБД (команда SQL или хранимая процедура).

Программа-тестер была написана на языке программирования Јava ${ }^{\mathrm{TM}}$ и имела простой интерфейс пользователя.

Он состоял из пяти полей ввода, трех кнопок, комбинированного списка, двух переключателей, а также трех таблиц, которые использовались аналогичным образом при работе, как с RESTful, так и SOAP Web-сервисом.

В тоже время программное обеспечение, использованное в данной работе, представляло собой последние версии соответствующих программных продуктов (на момент написания статьи). Оно содержало: пакет PHP 7.1.3, сервер Apache 2.4.25 и СУБД MySQL-5.7.17.

Приложения работали на персональном компьютере, частота центрального процессора которого была равна 1,2 ГГц, а объем оперативной памяти имел значение 8 ГБайт.

При подготовке к проведению исследований производительности разработанных приложений на сервер Арасhе устанавливались RESTful и SOAP Web-сервисы. Сервисы осуществляли поиск в таблицах Data1 - Data7.

B ходе измерений в программе STochno в списке "Service" выбирался тип Webсервиса (RESTful или SOAP), а переключателями "SQL" и "Procedure", устанавливался тип запроса к данным СУБД, после чего формировалась таблица "Request" с именами 
TECHNICAL SCIENCES AND TECHNOLOGIES

объектов исследования, а также максимальными значениями параметров поиска в них. С этой целью использовались поля ввода "Table" и "Мax", а также кнопка "Enter" интерфейса пользователя. Затем в поля ввода "URI" и "Reapet" вводились URI Webсервиса и число его обращений $N$ к каждой таблице при поиске в их данных, соответственно. В наших исследованиях $N=2000$.

Запуск тестирования осуществлялся нажатием на кнопку “Run” программы-тестера.

В процессе тестирования распределенное приложение работало с данными таблиц Data1 - Data7. При этом программа-тестер определяла значения полного времени выполнения запроса $\mathrm{T}$, времени обдумывания пользователя $t$, при формировании запроca, а также времени отклика $\tau$ Web-сервиса при поиске в данных. После чего в таблице “Times" приложения STochno появлялась информация о $\tau, t$ и T, а в таблице "Data", значение результата выполнения последнего запроса, при поиске в данных MySQL, для каждого объекта исследования.

C помощью кнопки “Save” результаты тестирования и его параметры сохранялись в текстовом файле. Имя файла для хранения данных предварительно вводилось в поле ввода "File".

На рис. 1 и 2 треугольниками представлены значения $\tau$, полученные при тестировании RESTful и SOAP PHP Web-сервисов. Черные треугольники - экспериментальные результаты для SOAP, белые - RESTful сервиса.

Как видно из этих рисунков, время отклика Web-сервисов, при поиске в данных MySQL с использованием, как команд SQL, так и хранимых процедур, возрастало при увеличении $V$. При этом, во всех рассматриваемых случаях, при десятикратном увеличении объема данных поиска, значения $\tau$ возрастали на величину $\cong 9 \cdot 10^{-3} \mathrm{c}$.

В тоже время экспериментальные значения времени отклика для SOAP сервиса превышали данные по $\tau$ для RESTful сервиса, полученные при тех же объемах $V$ и поиске с помощью как команд SQL, так и хранимых процедур, в среднем на $4 \cdot 10^{-3}$ с и $5 \cdot 10^{-3}$ с, соответственно.

Кроме того, использование разработанными программами хранимых процедур, вместо соответствующих команд SQL, приводило к росту $\tau$ на $2 \cdot 10^{-3}$ с для SOAP Webприложения и на $10^{-3}$ с для RESTful Web-службы.

Результаты тестирования были обработаны нами с помощью математической аппроксимации.

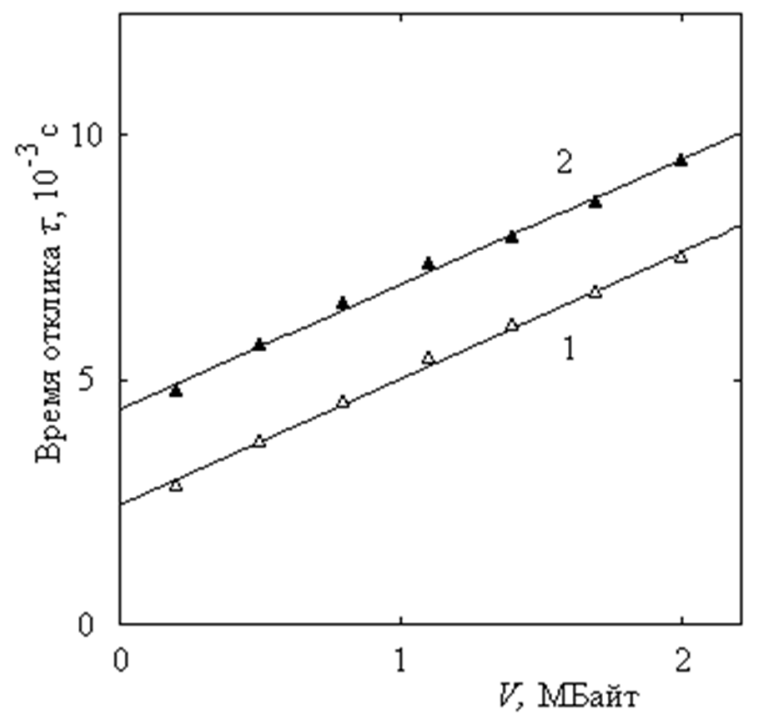

Puc. 1. Зависимости $\tau(V)$ для RESTful и SOAP PHP Web-сервисов при поиске в данных MySQL с использованием команд SQL (прямые 1 и 2 соответственно). Треугольники на рисункерезультаты тестирования 


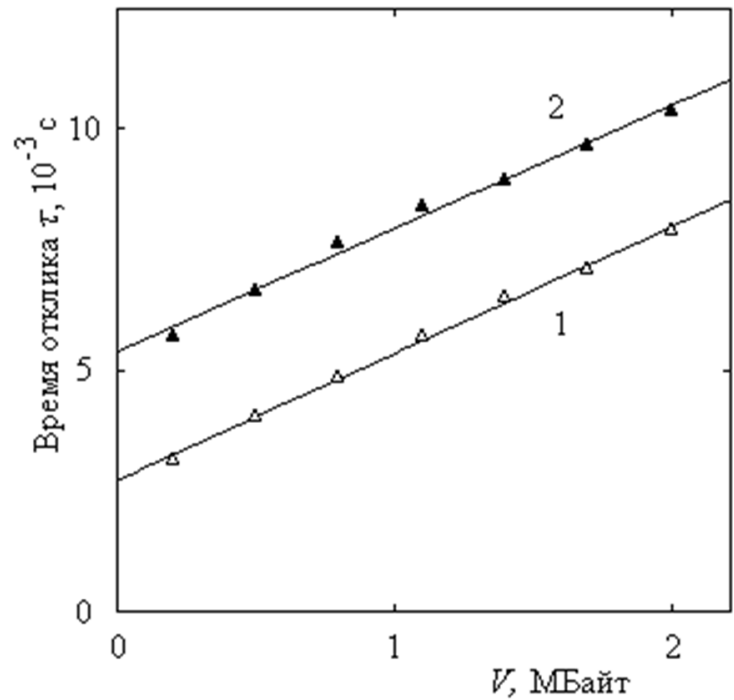

Pис. 2. Зависимости $\tau(V)$ для RESTful и SOAP PHP Web-сервисов при поиске в данных MySQL с использованием хранимых процедур (прямые 1 и 2 соответственно). Треугольники на рисунке - результаты тестирования

Для этих целей была использована дополнительная компьютерная программа, написанная нами на языке программирования Јava. Программа реализовывала метод наименьших квадратов [12] и позволяла, в частности, определять коэффициенты линейной регрессии и среднюю ошибку аппроксимации экспериментальных значений времени отклика.

При этом предполагалось, что время отклика $\tau$, для Web-сервисов при поиске в данных MySQL, может быть представлено в виде зависимости: $\tau=k \cdot V+b$, где $k$ и $b-$ постоянные коэффициенты.

Как выяснилось, при обработке результатов тестирования Web-сервисов с помощью метода наименьших квадратов, для всех исследованных режимов их функционирования, средняя ошибка аппроксимации экспериментальных значений времени отклика имела величину порядка 1,5 \%. При этом у функций регрессии $\tau(V)$ были положительные коэффициенты.

Зависимости $\tau(V)$, полученные в результате аппроксимации данных эксперимента, также приведены на рис. 1 и 2.

В тоже время, как приведенные на рис. 1 и 2 экспериментальные значения $\tau$, так и зависимости $\tau(V)$, полученные в результате их математической аппроксимации, указывают на то, что производительность RESTful Web-сервиса при поиске в данных MySQL с объемом $V \leq 2,0$ МБайт, была выше, чем у SOAP Web-службы, для всех исследованных режимов их функционирования.

Выводы и предложения. Таким образом, в данной работе нами был выполнен анализ производительности RESTful и SOAP PHP Web-сервисов, которые при поиске в данных MySQL в одном режиме функционирования использовали команды SQL, в другом - хранимые процедуры.

Как выяснилось, время отклика сервисов $\tau$ зависит от объема данных поиска $V$ и, при $V \leq 2.0$ Мбайт, для всех исследованных случаев удовлетворительно описывается линейными функциями $\tau(V)$ с положительными коэффициентами.

При этом применение в запросах к MySQL хранимых процедур, вместо команд $\mathrm{SQL}$, увеличивало значение времени отклика разработанных приложений и снижало их производительность.

В тоже время, при поиске в исследованных объемах данных $V$, с использованием при запросах к MySQL, как команд SQL, так и хранимых процедур, RESTful Webсервис был более производительным, чем сервис на основе SOAP. 
TECHNICAL SCIENCES AND TECHNOLOGIES

То есть для создания производительных PHP Web-сервисов для работы с данными MySQL целесообразно применять их реализацию на основе RESTful-технологии.

В дальнейшем предполагается провести подобные исследования с другими Webсерверами и СУБД. Кроме того, предполагается модернизировать разработанную программу-тестер для измерения производительности Web-сервисов при масштабируемых запросах.

\section{Список использованных источников}

1. Bean J. SOA and Web Services Interface Design: Principles, Techniques, and Standards / J. Bean. - Burlington, MA, USA: Elsevier Inc., 2010. - 360 p.

2. Горбенко A. В. Анализ особенностей создания и эксплуатации гарантоспособных сервис-ориентированных систем / А. В. Горбенко // Радіоелектронні і комп'ютерні системи. 2013. - № 5 (64). - C. 237-242.

3. Ernst M. D. Detection of Web Service substitutability and composability / M. D. Ernst, R. Lencevicius // Proceedings of the 1st International Workshop on Web Services Modeling and Testing (WS-MaTe 2006), June 2006, Palermo, Italy: proceedings. - 2006. - Pp. 123-135.

4. Harrison G. MySQL Stored Procedure Programming / G. Harrison, S. Feuerstein. - Sebastopol : O’Reilly Media, 2006. - 640 p.

5. Performance Comparison of Web Service Engines in PHP, Java, and C / T. Suzumura, S. Trent, M. Tatsubori, A. Tozawa, T. Onodera // Proceedings of the IEEE International Conference on Web Services (ICWS 2008), 23-26 September 2008, Beijing, China: proceedings. - 2008. Pp. 385-392.

6. Точилін С. Д. Продуктивність Java та PHP XML-RPC Web-служб пошуку в даних СКБД MySQL / С. Д. Точилин // Вісник ЖДТУ. - 2010. - № 1 (52). - С. 166-169.

7. Mitchell L. J. PHP Web Services / L. J. Mitchell. - Sebastopol, CA: O'Reilly Media, 2013. - 115 p.

8. Sklar D. PHP Cookbook / D. Sklar, A. Trachtenberg. - Sebastopol, CA: O'Reilly Media, 2014. $-820 \mathrm{p}$.

9. Menasce D. A. Capacity Planning for Web Services: metrics, models, and methods / D. A. Menasce, V. Almeida. - Upper Saddle River, NJ: Prentice Hall, 2001. - 608 p.

10. Welling L. PHP and MySQL Web development/ L. Welling, L. Thomson. - Indianapolis : Sams Publishing, 2005. $-946 \mathrm{p}$.

11. Гольиман В. И. MySQL 5.0. Библиотека программиста / В. И. Гольцман. - СПб. : Питер, 2010. $-253 \mathrm{c}$.

12. Кремер Н. Ш. Теория вероятностей и математическая статистика / Н. Ш. Кремер. - М. : ЮНИТИ-ДАНА, 2002. - 543 с.

\section{References}

1. Bean, J. (2010). SOA and Web Services Interface Design: Principles, Techniques, and Standards. Burlington, MA, USA: Elsevier Inc.

2. Gorbenko, A.V. (2013). Analiz osobennostei sozdaniia i ekspluatatsii garantosposobnykh servis-orientirovannykh sistem [Analysis of dependable service-oriented systems development features]. Radioelektronni i kompiuterni systemy - Radioelectronic and computer systems, no. 5 (64), pp. 237 242 (in Russian).

3. Ernst, M.D., Lencevicius, R. (2006). Detection of Web Service substitutability and composability. Proceedings from 1st International Workshop on Web Services Modeling and Testing (WSMaTe 2006). (Italy, Palermo, June, 2006), pp. 123-135.

4. Harrison, G., Feuerstein S. (2006). MySQL Stored Procedure Programming. Sebastopol: O’Reilly Media.

5. Suzumura, T., Trent, S., Tatsubori, M., Tozawa, A., Onodera, T. (2008). Performance Comparison of Web Service Engines in PHP, Java, and C. Proceedings from IEEE International Conference on Web Services (ICWS 2008). (China, Beijing, September 23-26, 2008), pp. 385-392.

6. Tochilin, S.D. (2010). Produktyvnist Java ta PHP XML-RPC Web-sluzhb poshuku v danykh SKBD MySQL [Productivity of Java and PHP Web services of search in the DBMS MySQL data]. Visnyk Zhytomyrskoho derzhavnoho universytetu - The journal of zhytomyr state technological university, no. 1(52), pp .166-169 (in Ukrainian).

7. Mitchell, L.J. (2013). PHP Web Services. Sebastopol, CA: O'Reilly Media.

8. Sklar, D., Trachtenberg, A. (2014). PHP Cookbook. Sebastopol, CA: O'Reilly Media. 
9. Menasce, D.A., Almeida, V. (2001). Capacity Planning for Web Services: metrics, models, and methods. Upper Saddle River, NJ: Prentice Hall.

10. Welling, L., Thomson, L. (2005). PHP and MySQL Web development. Indianapolis: Sams Publishing.

11. Goltzman, V.I. (2010). MySQL 5.0. Biblioteka programmista [MySQL 5.0. Library of the programmer]. Saint-Petersburg: Piter (in Russian).

12. Kremer N.S. (2002). Teoriia veroiatnostei i matematicheskaia statistika [Theory of probabilities and mathematical statistics]. Moscow: UNITI-DANA (in Russian).

\section{Sergiy Tochilin \\ PERFORMANCE OF RESTfUI AND SOAP PHP WEB SERVICES FOR DATA SEARCH IN MYSQL}

Urgency of the research. The service oriented architecture (SOA) is widely used in terms of constructing distributed computer systems. Web service technologies are applied for practical implementation of the system with SOA.

One of the essential requirements to Web services is the performance of functioning, which depends on their software implementation.

Target setting. Web services are used to access information stored in DBMS databases posted on websites.

The issue of optimum software selection for Web service implementation of information with real-time data access is urgent in this connection.

Actual scientific researches and issues analysis. While accessing data, Web services use inquiries to DBMS, which are usually implemented as SQL commands.

In order to enhance the efficiency of operation of Web applications working with data, stored procedures and functions of DBMS are used instead of SQL commands.

Uninvestigated parts of general matters. No investigations of performance of RESTful and SOAP PHP Web services that during the access to MYSQL data with different volumes of stored information used SQL commands in one function mode and stored procedures in another one were conducted.

The research objective. To define and analyze the performance of RESTful and SOAP PHP Web services using SQL commands and functionally similar stored procedures for the search of MySQL data for the selection of optimum software of Web services working with MySQL DBMS.

The statement of basic materials. As it turned out, the use of stored procedures instead of SQL commands by RESTful and SOAP PHP Web services when inquiring to MySQL for the search of data reduced their performance.

At the same time while searching in the investigated volumes of data $V \leq 2$ MByte, when applying both SQL commands and stored procedures in inquiring to MySQL, RESTful Web service was more performing than SOAP PHP service.

Conclusions. The particularities of performance of RESTful and SOAP PHP services when searching in MySQL data with the use of both SQL commands and stored procedures have been established. While searching in MySQL data, RESTful Web service was more performing in all functioning modes than SOAP PHP service.

Key words: performance, SOAP, RESTful, Web service, MySQL.

\section{Сергій Точилін}

\section{ПРОДУКТИВНІСТЬ RESTfUI TA SOAP PHР WЕВ-CЕРВICIB ПРИ ПОШУКУ В ДАНИХ МУSQL}

Розроблені RESTful i SOAP PHP Web-сервіси, які використовують команди SQL та збережені проиедури при запитах до MySQL на пошук у даних, а також Јаvа програма-тестер для визначення їхньої продуктивності. 3 допомогою створених програм отримані дані про продуктивність Web-сервісів при обробиі різних обсягів інформачії, яка зберігається в базі даних MySQL. Результати досліджень, отримані для RESTful ma SOAP Web-сервісів, вказують на більш високу продуктивність RESTful сервісу при пошуку в даних MySOL з використанням як команд SOL, так $i$ збережених процедур. У той час при звертанні Web-сервісів до даних MySQL з використанням збережених прочедур, замість команд SQL, їхньої продуктивність знижувалася.

Ключові слова: продуктивність; SOAP; RESTful; Web-сервic; MySQL.

Точилин Сергей Дмитриевич - кандидат физико-математических наук, доцент, доцент кафедры компьютерных систем и сетей, Запорожский национальный технический университет (ул. Жуковского, 64, г. Запорожье, 69093, Украина).

Точилін Сергій Дмитрович - кандидат фізико-математичних наук, доцент, доцент кафедри комп’ютерних систем та мереж, Запорізький національний технічний університет (вул. Жуковського, 64, м. Запоріжжя, 69093, Україна).

Tochilin Sergiy - PhD in Physico-Mathematical Sciences, Associate Professor, Associate Professor of Department of Computer Systems and Networks, Zaporizhzhya National Technical University (64 Zhukovsky Str., 69063 Zaporizhzhia, Ukraine).

E-mail: tochnozp@gmail.com

Scopus Author ID: 6602607112

Точилин С. Производительность RESTful и SOAP PHP WEB-сервисов при поиске в данных MYSQL / С. Точилин // Технічні науки та технології. - 2017. - № 2 (8). - С. 116-122. 